\title{
The course of skull deformation from birth to 5 years of age: a prospective cohort study
}

\author{
Leo A van Vlimmeren ${ }^{1,2}$ - Raoul HH Engelbert ${ }^{3,4}$ - Maaike Pelsma ${ }^{1,2}$. \\ Hans MM Groenewoud ${ }^{5}$ • Magda M Boere-Boonekamp ${ }^{6}$. \\ Maria WG Nijhuis-van der Sanden ${ }^{1,2}$
}

Received: 24 February 2016 /Revised: 17 August 2016 / Accepted: 18 October 2016 / Published online: 4 November 2016

(C) The Author(s) 2016. This article is published with open access at Springerlink.com

\begin{abstract}
In a continuation of a prospective longitudinal cohort study in a healthy population on the course of skull shape from birth to 24 months, at 5 years of age, 248 children participated in a follow-up assessment using plagiocephalometry (ODDI—oblique diameter difference index, CPI—cranio proportional index). Data from the original study sampled at birth, 7 weeks, 6,12 , and 24 months were used in two linear mixed models. Main findings: (1) if deformational plagiocephaly (ODDI <104\%) and/or positional preference at 7 weeks of age are absent, normal skull shape can be predicted at 5 years of age; (2) if positional preference occurs,
\end{abstract}

Electronic supplementary material The online version of this article (doi:10.1007/s00431-016-2800-0) contains supplementary material, which is available to authorized users.

Leo A van Vlimmeren

leo.vanvlimmeren@radboudumc.nl

Raoul HH Engelbert

r.h.h.engelbert@hva.nl

Maaike Pelsma

maaike.pelsma@radboudumc.nl

Hans MM Groenewoud

hans.groenewoud@radboudumc.nl

Magda M Boere-Boonekamp

m.m.boere-boonekamp@utwente.nl

Maria WG Nijhuis-van der Sanden

ria.nijhuis-vandersanden@ radboudumc.nl

1 Department of Rehabilitation, Paediatric Physical Therapy, Radboud university medical center, P.O. 9101, 6500

HB Nijmegen, The Netherlands
ODDI is the highest at 7 weeks and decreases to a stable lowest value at 2 and 5 years of age; and (3) regarding brachycephaly, all children showed the highest CPI at 6 months of age with a gradual decrease over time.

Conclusion: The course of skull deformation is favourable in most of the children in The Netherlands; at 5 years of age, brachycephaly is within the normal range for all children, whereas the severity of plagiocephaly is within the normal range in $80 \%$, within the mild range in $19 \%$, and within the moderate/severe range in $1 \%$. Medical consumption may be reduced by providing early tailored counselling.

2 Radboud Institute for Health Sciences, IQ healthcare, Radboud university medical center, P.O. 9101, 6500

HB Nijmegen, The Netherlands

3 ACHIEVE, Center of Applied Research, Faculty of Health, University of Applied Sciences, Tafelbergweg 51, 1000

CN Amsterdam, The Netherlands

4 Academic Medical Center, Department of Rehabilitation, University of Amsterdam, Meibergdreef 9, 1105

AZ Amsterdam, The Netherlands

5 Department for Health Evidence, Radboud university medical center, P.O. 9101, 6500 HB Nijmegen, The Netherlands

6 Department Health Technology and Services Research, Institute for Governance Studies, University of Twente, P.O. 217, 7500

AE Enschede, The Netherlands 


\section{What is Known:}

- Skull deformation prevalence increased after recommendations against Sudden Infant Death Syndrome, little is known about the longitudinal course.

- Paediatric physical therapy intervention between 2 and 6 months of age reduces deformational plagiocephaly at 6 and 12 months of age.

What is New:

- The course of skull deformation is favourable in most of the children in The Netherlands; at 5 years of age, deformational brachycephaly is within the normal range for all children, whereas the severity of deformational plagiocephaly is within the normal range in $80 \%$, within the mild range in 19\%, and within the moderate to severe range in only $1 \%$.

- Paediatric physical therapy intervention does not influence the long-term outcome; it only influences the earlier decrease of the severity of deformational plagiocephaly.

Keywords Deformational brachycephaly $\cdot$ Deformational plagiocephaly $\cdot$ Newborns $\cdot$ Skull deformation

\author{
Abbreviations \\ CPI Cranio proportional index \\ DB Deformational brachycephaly \\ DP Deformational plagiocephaly \\ ODDI Oblique diameter difference index \\ PCM Plagiocephalometry \\ PPT Paediatric physical therapy intervention
}

\section{Introduction}

Since epidemiological studies have showed that prone and side sleeping were major risk factors for sudden infant death syndrome (SIDS) [12, 14], supine sleeping has increased, consistent with the recommendations of the American Academy of Paediatrics [2, 3, 13, 15]. Simultaneously, a prevalence increase of skull deformation has also been observed $[4,6,7,19,29]$; asymmetrically, this is described as deformational plagiocephaly (DP) and/or symmetrically, which is described as deformational brachycephaly (DB) [4, 19, 32, 34].

The prevalence of DP and DB increases rapidly in young children during the first months of life [19, 21, $34,39]$. DP is attributed to perinatal factors [16, 21, 26, $34,39]$ as well as factors in early infancy $[6,17,19,39]$. Familial and ethnic factors are supposed to be related to skull deformations [25, 31, 34]. Positional preference, when children lie on their back, is the major cause of these skull deformations [4, 7, 19, 34]; children keep their head turned with the same spot on the surface, which slows down growth in that direction and stimulates growth in the other directions [4, 7, 18, 34]. Many clinicians consider skull deformation to be a minor and purely cosmetic condition $[11,20]$. Hutchison et al. reported that $4 \%$ of skull deformations remained severe at 3 to 4 years of age [20]. In a cross-sectional study, Roby et al. found a prevalence of DP of $1 \%$ and DB of $1.1 \%$ in 15 -year-old teens [33]. Of these children with DP or DB, $38.1 \%$ was noted to have abnormal facial characteristics [33].

In children with DP and DB, several conservative interventions are applied: (paediatric) physical therapy [5, 9, 43], helmet therapy [18, 24, 27, 32, 36, 37, 46], manual therapy [8], osteopathy [35], and surgical intervention [22, 30].

The aim of the present study was to investigate the longterm course of skull shape in healthy newborns until the age of 5.5 years, with special interest in the subgroups of children with and without positional preference at 7 weeks, and in the children with positional preference who received paediatric physical therapy intervention (PPT) or not (no PPT).

\section{Methods}

This study provides additive follow-up data of a prospective cohort study with an embedded randomised controlled trial to assess the effect of paediatric physical therapy, with measurements at birth, 7 weeks, 6 and 12 months of age. The additive data of the measurements in children at 2 and 5.5 years of age are presented in this article.

\section{Participants}

The original prospective cohort study started with 380 healthy newborns ( $\geq 36$-weeks gestation), born between December 2004 and September 2005 at the general district hospital Bernhoven in Veghel, The Netherlands. Children with congenital muscular torticollis (torticollis with a one-sided shortening of the sternocleidomastoid muscle; Kaplan type 2 and 3 [23, 39, 40]), dysmorphism, or syndromes were excluded. A flowchart of included and excluded children over time is presented in Fig. 1. At 7 weeks of age, the embedded randomised controlled trial started and the cohort of children was divided into three groups: (1) children without positional preference $(n=315)$, (2) children with positional preference $(n=65)$ and randomly allocated to PPT $(n=33)$, and (3) children with positional preference and randomly allocated to no PPT $(n=32)$. Results of the RCT until the age of 12 months are presented elsewhere: PPT between 2 and 6 months of age was established to be effective in children with positional preference in reducing DP at 6 and 12 months of age $[42,43]$. Therefore, we decided to present the long-term outcome at 2 and 5.5 years for the three above-mentioned subgroups. 
Fig. 1 Flowchart of the children assessed six times from birth to 5.5 years of age

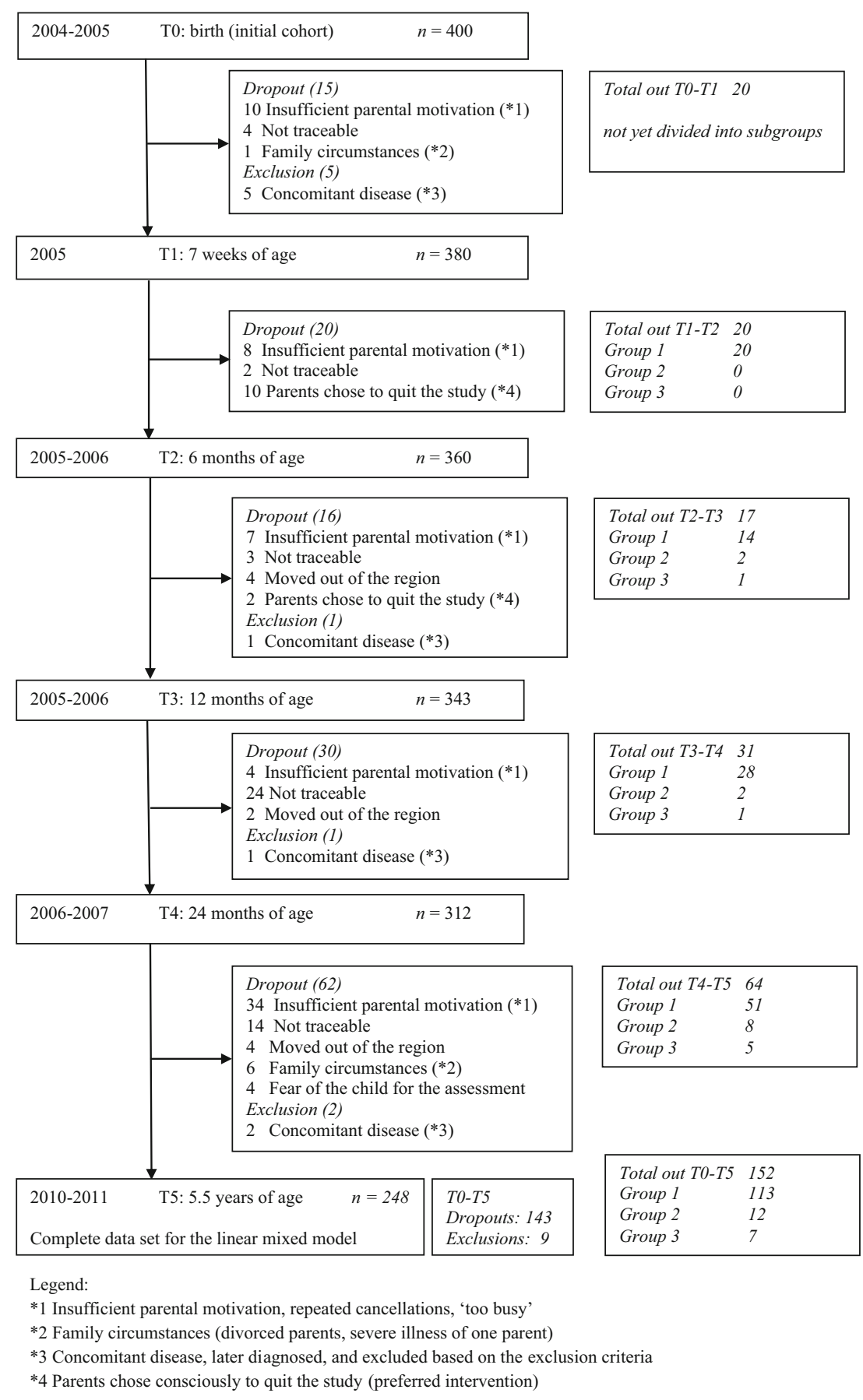

\section{Measures}

Participating children were measured at birth (T0), 7 weeks (T1), 6 months (T2), and 12 months (T3). Long-term outcome data were collected at 24 months (T4) and 5.5 years (T5) of age.

\section{General characteristics and risk factors}

General characteristics including gender, birth rank, parental age, parental educational level, and obstetric data including gestation, pregnancy rank, presentation at delivery, mode of delivery, length of labour, multiple birth, Apgar score, birth weight, and birth head circumference were collected within $48 \mathrm{~h}$ of birth.

Gender, being firstborn, nursing, feeding, sleeping, and playing positioning habits (positional preference when sleeping, head to the same side on a chest of drawers, only bottle-feeding, positioning to the same side during bottle-feeding, 'tummy time' when awake $<3$ times per day, and slow achievement of motor milestones with the 
presence of DP) were considered as potential risk factors, based on a previous study [42].

\section{Primary outcome measure}

The transversal shape of the skull was measured at all six assessments (T0-T5) by plagiocephalometry (PCM), which is a reliable, valid and responsive instrument [27, 38, 41]. PCM measures the relationship between the transverse shape of the skull and the position of both ears and nose, and thereby the location and amount of flattening of the skull. PCM assesses the severity of DP by the parameter oblique diameter difference index (ODDI: ratio between both oblique diameters of the head) and the severity of DB by the parameter cranio proportional index (CPI: ratio between the width and length of the head). Based on psychometric analysis in a previous study [41] and analogous to other relevant studies [44, 45], we showed that clinically relevant asymmetrical (DP) skull flattening was present in the case of ODDI $\geq 104 \%$, and symmetrical (DB) skull flattening was present in the case of CPI $\geq 90 \%$. Furthermore, we defined four categories of skull deformation, whereas ODDI refers to DP, and CPI refers to DB: (1) normal: ODDI <104 and/or CPI <90, (2) mild: ODDI 104-107 and/or CPI 90-94, (3) moderate: ODDI 108-111 and/or CPI 95-99, and (4) severe: ODDI $\geq 112$ and/or $\mathrm{CPI} \geq 100$.

PCM was performed by two very experienced examiners who were blinded for the group belonging and who were interchangeable (LV author, FG acknowledgements) [41-43]. The environmental conditions (temperature, light, positioning) during the assessments were the same for all children.

\section{Paediatric physical therapy intervention}

In 65 children with positional preference, PPT was indicated as described previously [43]. In 33 children, a standardised PPT program was executed due to randomisation of the study between 2 and 6 months of age. The PPT program consisted of exercises to reduce positional preference and to stimulate motor development, by counselling parents on positioning, handling and nursing, supported by a leaflet with basic preventive advice. PPT was stopped when the positional preference no longer occurred during the day and night, when awake and asleep, when the parents were shown to have incorporated all of the advice and exercise in daily handling, and when there were no indications for motor developmental problems (delays or asymmetries). The parents of the control group (no PPT) received only a leaflet with basic preventive advices, without further education to intervene. Both groups received regular advice from well-baby clinics, like every child in The Netherlands [43].

\section{Statistics}

Descriptive statistics were used to analyse baseline characteristics. Means and standard deviations or proportions were calculated for the relevant variables. In the present study, we assessed the association between peri- and postnatal factors, and skull deformation data at 7 weeks of age with the skull deformation at 24 months and 5.5 years of age. The relationship between these factors and deformity was analysed by means of cross-tabulation, as well as linear and logistic regression. In the univariate analyses, putative risk factors with a $P<0.15$ were selected for inclusion in multivariate models. In the linear regression analysis, the effect of these factors on the dependent factors ODDI (continuous) and CPI (continuous) was assessed at 24 months and 5.5 years of age.

To describe the primary outcome measures ODDI and CPI, two linear mixed models were constructed. One model had ODDI as the dependent variable (related to DP) and the other had CPI as the dependent variable (related to DB). We included time, positional preference at 7 weeks of age and the outcome measures ODDI and CPI at birth as independent variables. The models with the three subgroups, as illustrated in the design, included interactions between positional preference and measurements in time. This time-by-positional preference interaction showed whether there was a difference between the groups over the study period. Time, positional preference at 7 weeks of age, independent ODDI or CPI variables at birth, and the interaction term between positional preference and time were all entered in the models as fixed factors. The ODDI pattern is based on a chosen ODDI at birth of 101. The CPI pattern is based on a chosen CPI at birth of 79 .

Residual plots from the mixed models were examined to check model assumptions. Both linear mixed model analyses were performed on the three subgroups.

The mean (95\% confidence interval) ODDI or CPI were computed at each time point for each group for a given value of the ODDI and CPI at birth. These parameters also enabled us to estimate the difference between the three positional preference groups at each time point, corrected for the score of the dependent variable at birth. Although not all parameters in both models showed a significant difference from 0 , we kept all variables in the model for reasons of consistency.

Analyses were executed as two-tailed with a significance level of $5 \%$. When applicable, $95 \%$ confidence intervals were computed. Statistical analyses were performed using SAS software version 9.2 and IBM SPSS Statistics 20.0 software. 


\section{Results}

\section{General characteristics of the study population}

We included 380 healthy newborns in the cohort and assessed them shortly after birth. Of these, 248 children $(65 \%)$ with a mean age of 5.51 years (standard deviation 0.19 years) were analysed at T5: there were 202 children without positional preference, 21 children with positional preference allocated to PPT, and 25 children with positional preference allocated to no PPT. General characteristics of the three included groups at $\mathrm{T} 1$ $(n=380)$ and relevant determinants for DP and DB at later age are presented in Table 1.

The reasons for dropouts and exclusion are illustrated in Fig. 1. Independent $T$ tests showed no significant differences in gender and positioning, as well as in ODDI and CPI per subgroup, at each of the earlier time points between participants at T5 and children who dropped out before T5. No other treatments, except PPT in the intervention group, were applied.

\section{Risk factors identified at 7 weeks of age}

There was no association between the potential risk factors nursing, feeding, sleeping, and playing positioning habits at 7 weeks of age and skull deformity at 24 months and 5.5 years of age. At 24 months of age, there was a univariate association between the time spent playing prone ('tummy time') measured at 7 weeks of age and the ODDI percentage $(\beta=-0.304$, $P=0.062,95 \% \mathrm{CI}=-0.624$ to 0.015$)$. In the univariate analyses, no putative risk factors with a $P<0.15$ could be found to be associated with the PCM measurements at 5.5 years of age.

\section{Primary outcome}

The courses of DP and DB over time in the three groups are illustrated in Table 2 and Figs. 2 and 3. Skull deformation regarding DP at T5 in children without positional preference occurred in $17.3 \%$ (35 out of 202) at T5. None of the children without positional preference showed DB at T5. In the PPT group, 8 out of $21(38 \%)$ and in the no PPT group, 8 out of 25

Table 1 General characteristics $(n=380)$ of the three included groups at T1 (7 weeks of age. Data are presented as $n(\%)$ or mean (standard deviation [SD])

\begin{tabular}{|c|c|c|c|c|c|c|c|c|c|}
\hline & \multicolumn{3}{|c|}{$\begin{array}{l}\text { Group } 1 \\
\text { No positional preference } \\
(n=315)\end{array}$} & \multicolumn{3}{|c|}{$\begin{array}{l}\text { Group } 2 \\
\text { Positional preference and randomly } \\
\text { allocated to PPT }(n=33)\end{array}$} & \multicolumn{3}{|c|}{$\begin{array}{l}\text { Group } 3 \\
\text { Positional preference and randomly } \\
\text { allocated to no PPT }(n=32)\end{array}$} \\
\hline & $n$ & $\%$ & & $n$ & $\%$ & & $n$ & $\%$ & \\
\hline Gender, boy & 138 & 43.8 & & 20 & 60.6 & & 20 & 62.5 & \\
\hline First pregnancy & 112 & 35.6 & & 16 & 48.5 & & 14 & 43.8 & \\
\hline \multicolumn{10}{|l|}{ Delivery } \\
\hline Vaginal & 205 & 65.1 & & 23 & 69.7 & & 19 & 59.4 & \\
\hline Vacuum-assisted & 35 & 11.1 & & 4 & 12.1 & & 4 & 12.5 & \\
\hline Caesarean section & 75 & 23.8 & & 6 & 18.2 & & 9 & 28.1 & \\
\hline \multicolumn{10}{|l|}{ Birth rank } \\
\hline First born & 141 & 44.8 & & 17 & 52 & & 16 & 50.0 & \\
\hline Later born & 174 & 55.2 & & 16 & 48.5 & & 16 & 50.0 & \\
\hline \multicolumn{10}{|l|}{ Tummy time till 7 weeks of age (T1) } \\
\hline$<5$ min per session & 206 & 65.4 & & 26 & 78.8 & & 25 & 78.1 & \\
\hline 5 to 15 min per session & 75 & 23.8 & & 5 & 15.2 & & 5 & 15.6 & \\
\hline \multirow[t]{2}{*}{$>15$ min per session } & 34 & 10.8 & & 2 & 6.1 & & 2 & 6.3 & \\
\hline & $n$ & Mean & SD & $n$ & Mean & SD & $n$ & Mean & SD \\
\hline \multicolumn{10}{|l|}{ Age (years) at birth from } \\
\hline Mother & 315 & 31.1 & 4.30 & 33 & 30.2 & 3.35 & 32 & 31.4 & 3.88 \\
\hline Father & 311 & 33.8 & 4.87 & 33 & 33.7 & 4.97 & 32 & 33.6 & 5.03 \\
\hline Skull circumference at birth $(\mathrm{cm})$ & 315 & 34.7 & 1.44 & 33 & 35.2 & 1.37 & 32 & 34.9 & 1.17 \\
\hline Birth weight (kg) & 315 & 3.3 & 0.48 & 33 & 3.5 & 0.44 & 32 & 3.5 & 0.45 \\
\hline Gestation (weeks) & 315 & 39.4 & 1.48 & 33 & 39.7 & 1.46 & 32 & 39.5 & 1.43 \\
\hline Length of labour, second stage (hours) & 242 & 0.51 & 0.47 & 28 & 0.62 & 0.59 & 24 & 0.65 & 0.61 \\
\hline
\end{tabular}

$P P T$ paediatric physical therapy intervention 
(32\%) children with positional preference still showed DP at T5. Only two children with positional preference showed DB at T5: mild DB was reported in the no PPT group.

\section{Course of DP}

The predictive model for ODDI showed a significant interaction for the parameter time point $(P<0.0001)$, positional preference $(P<0.0001)$, and their interaction $(P<0.0001)$, but not for ODDI at birth $(P=0.55)$. Therefore, the ODDI at birth did not influence the value of ODDI at later time points. The group without positional preference showed an almost stable ODDI over time. Both groups with positional preference showed a strong increase of ODDI at 7 weeks of age and then a gradual decrease over time. However, in children allocated to PPT, the decrease was earlier than in children without intervention, as shown by a significant interaction effect at 6 and 12 months of age. The differences between groups 2 and 3 are small. The outcome of the linear mixed model analysis of the prospective ODDI (DP) is demonstrated in Fig. 2 and Table 3.

\section{Course of $D B$}

The predictive model for CPI showed a significant interaction for the parameter time point $(P<0.0001)$, positional preference $(P<0.001)$, and CPI at birth $(P<0.0001)$, but not for the interaction term between time and positional preference $(P=0.13)$. CPI at later time points was strongly influenced by the CPI at birth. There was a change in CPI over time, but the pattern was the same for all groups: an increase in CPI at 6 months, followed by a slow decrease to values comparable to the initial values at birth. The group without positional preference had lower scores at all of the time points. Overall the differences between groups are small (Fig. 3 and Table 4).

\section{Discussion}

This is the first prospective study of the course of skull shape in healthy newborns with a 5.5-year follow-up. The course of skull deformation is favourable; at 5.5 years of age, CPI is within the normal range for all children, whereas the course of plagiocephaly differs: $80 \%$ of the ODDI scores are within the normal range, $19 \%$ in the mild range, and only $1 \%$ in the moderate to severe range. About $20 \%$ of the children scored outside the normal range, which seems to be clinically relevant.

Children with positional preference and DP allocated to PPT showed a rapid decrease of DP, measured at 6 and 12 months, but did not decrease further at 2 and 5.5 years. Remarkably, the children with positional preference and DP allocated to the no PPT group showed a similar result for DP 
Fig. 2 Patterns of the predicted mean ODDI and its $95 \%$ confidence interval for the three subgroups

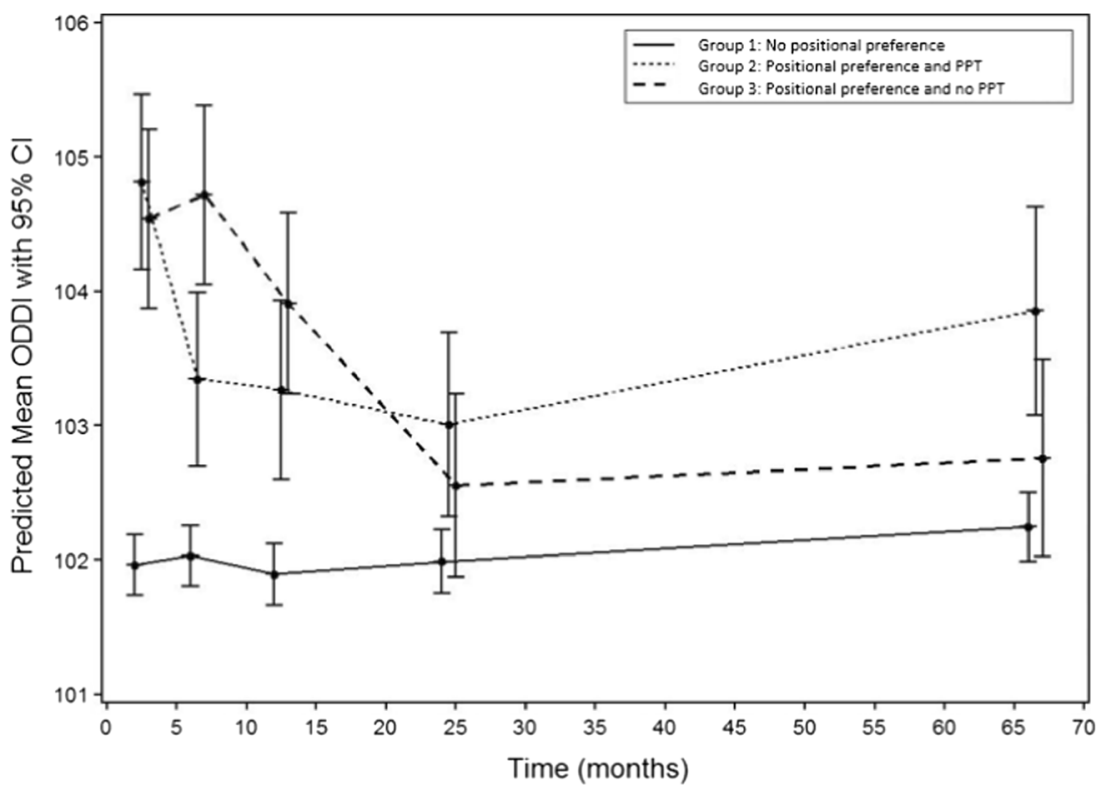

measured at 2 and 5.5 years of age. Overall, the mean DB at 5.5 years of age more or less reached the values of the initial means of DB at birth.

The strengths of the study are the prospective design starting with a healthy population of newborns, the embedded randomised controlled trial regarding the effects of PPT and the use of a reliable and valid primary outcome measurement (plagiocephalometry (PCM)) [38, 42-45]. Also, the use of the (multi-level) linear mixed model analyses of the follow-up data provides a realistic view on the course of skull development and deformation.

This study has several limitations. The definition of muscular torticollis differs in the international literature, which might be confusing in interpreting and comparing studies on asymmetry in infancy. Kaplan et al. [23] categorised congenital muscular torticollis (CMT) into three types: (1) postural CMT presents as the infant's postural preference but without muscle or passive ROM restrictions and is the mildest form; (2) muscular CMT presents with sternocleidomastoid muscle tightness and passive ROM limitations; and (3) SCM mass CMT, the most severe form, presents with a fibrotic thickening of the sternocleidomastoid muscle and passive ROM limitations. In The Netherlands, the entity of congenital muscular torticollis concerns Kaplan CMT types 2 and 3, and not type 1, which concerns the ordinary postural torticollis [23, 39-45]. In our study, we included type 1 and excluded types 2 and 3.

In the initial study, PPT was performed between 2 and 6 months of age in the intervention group. Having had PPT probably plays a minor role in the development of the skull in
Fig. 3 Patterns of the predicted mean CPI and its $95 \%$ confidence interval for the three subgroups

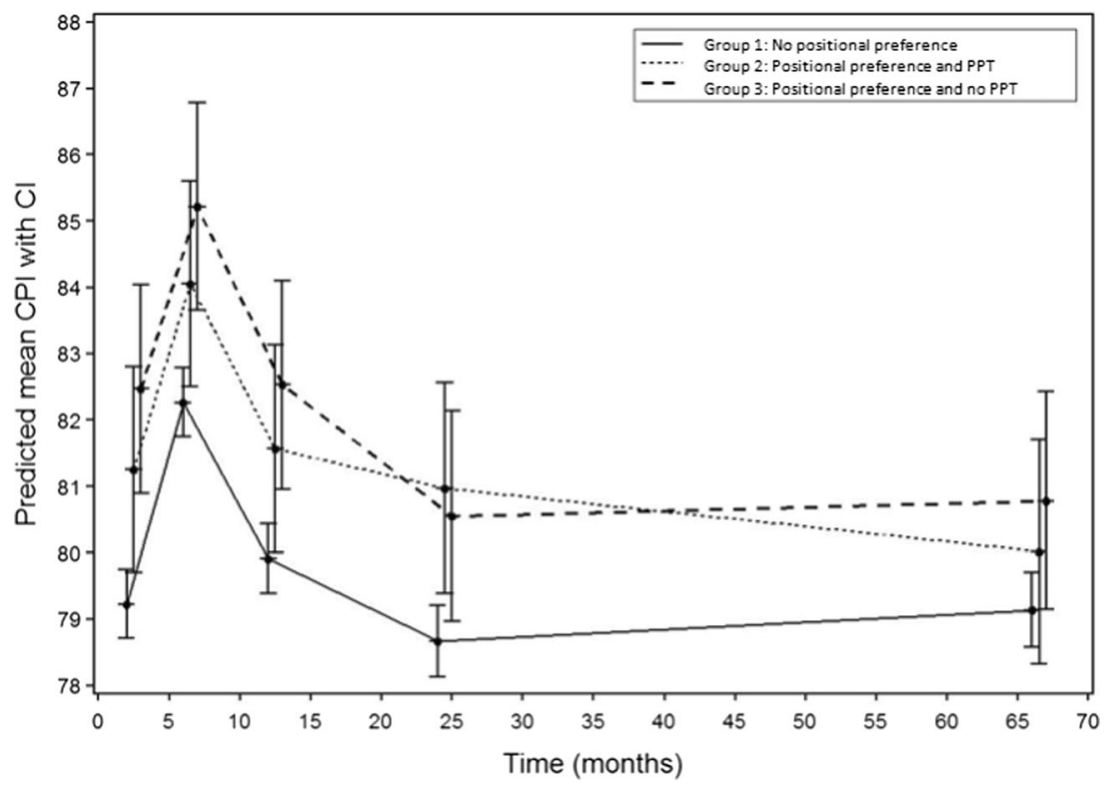


Table 3 Estimated group differences for ODDI at 7 weeks, 6 months, 24 months and 5.5 years

\begin{tabular}{llc}
\hline Estimated difference & Group differences (with 95\% CI) & $P$ value \\
\hline Mean ODDI group 3 - mean ODDI group 1 & $2.58(1.88 ; 3.27)$ & $<0.0001$ \\
7 weeks of age & $2.69(1.99 ; 3.38)$ & $<0.0001$ \\
6 months of age & $2.01(1.31 ; 2.72)$ & $<0.0001$ \\
12 months of age & $0.57(-0.15 ; 1.28)$ & 0.12 \\
24 months of age & $0.51(-0.26 ; 1.28)$ & 0.19 \\
5.5 years & & 0.56 \\
Mean ODDI group 3 - mean ODDI group 2 & $-0.27(-1.19 ; 0.65)$ & 0.004 \\
7 weeks of age & $1.38(0.45 ; 2.30)$ & 0.18 \\
6 months of age & $0.64(-0.30 ; 1.58)$ & 0.36 \\
12 months of age & $-0.45(-1.41 ; 0.51)$ & 0.04 \\
24 months of age & $-1.10(-2.16 ;-0.04)$ & $<0.0001$ \\
5.5 years & & 0.0002 \\
Mean ODDI group 2 - mean ODDI group 1 & $2.85(2.17 ; 3.54)$ & 0.0001 \\
7 weeks of age & $1.32(0.63 ; 2.00)$ & 0.006 \\
6 months of age & $1.37(0.67 ; 2.07)$ & 0.0001 \\
12 months of age & $1.02(0.30 ; 1.74)$ & \\
24 months of age & $1.61(0.79 ; 2.42)$ & \\
5.5 years & &
\end{tabular}

the following 5 years. The effect of PPT on skull shape appears to disappear at 24 months of age; therefore, it might have been better to have used PCM outcome to randomise to intervention. When we developed and constructed the initial study, we used the hypothetical rationale that positional preference always occurs before skull deformation. Also, of course, skull deformation remains longer, even when positional preference has disappeared.
From the initial cohort of 380 children, 152 children were lost to follow-up. The general characteristics and PCM measurement values of the children who left the study at each of the time points before T5 were compared with the children who remained in the study. We analysed and compared the skull measurement characteristics at every time point before the last measurement, when the (later) lost to follow up children were still in the longitudinal cohort and were measured.
Table 4 Estimated group differences for CPI at 7 weeks, 6 months, 24 months, and 5.5 years

\begin{tabular}{lll}
\hline Estimated difference & Group differences (with 95\% CI) & $P$-value \\
\hline Mean CPI group 3 - mean CPI group 1 & $3.24(1.59 ; 4.89)$ & 0.0001 \\
7 weeks of age & $2.94(1.29 ; 4.59)$ & 0.0005 \\
6 months of age & $2.62(0.96 ; 4.28)$ & 0.002 \\
12 months of age & $1.88(0.21 ; 3.55)$ & 0.027 \\
24 months of age & $1.64(-0.09 ; 3.38)$ & 0.063 \\
5.5 years & & 0.28 \\
Mean CPI group 3 - mean CPI group 2 & $1.21(-0.99 ; 3.42)$ & 0.30 \\
7 weeks of age & $1.16(-1.04 ; 3.37)$ & 0.39 \\
6 months of age & $0.96(-1.26 ; 3.18)$ & 0.71 \\
12 months of age & $-0.42(-2.67 ; 1.82)$ & 0.52 \\
24 months of age & $0.77(-1.58 ; 3.12)$ & \\
5.5 years & & 0.015 \\
Mean CPI group 2 - mean CPI group 1 & $2.02(0.40 ; 3.66)$ & 0.032 \\
7 weeks of age & $1.78(0.15 ; 3.41)$ & 0.049 \\
6 months of age & $1.66(0.01 ; 3.30)$ & 0.007 \\
12 months of age & $2.30(0.63 ; 3.97)$ & 0.34 \\
24 months of age & $0.87(-0.91 ; 2.65)$ & \\
5.5 years & &
\end{tabular}


Therefore, we expect that the children lost to follow up did not bias the outcomes, but this can be discussed, because there can be other reasons why parents did not meet the invitation for the final measurement. The dropout percentages differ between groups, but the differences between group 2 (PPT) and group 3 (no PPT) are very small and acceptable.

A limitation discussion point is the use of the cut-off points of ODDI $\geq 104 \%$ for DP and of CPI $\geq 90 \%$ for DB. Some authors discussed the use of cut-off points in skull deformation and it is obvious that the use of other cut-offs will provide other prevalences of severity $[19,20]$. The cut-off points were based on a statement in the plagiocephalometry reliability study [41] and were similar to the plagiocephalometry cutoff points used in another recent intervention study regarding skull deformations (HEADS helmet study) [44, 45]. Also, the dichotomy of DP and DB can be discussed. Meyer-Marcotty et al. suggested using a continuum rather than differentiating between the presence or absence of skull deformation, because of the overlapping criteria of DP and DB [28].

Although the course of skull deformation in newborns seems to be favourable, not all of the children with DP (ODDI $\geq 104 \%$ ) at 24 months of age fully recovered at 5.5 years of age. At 5.5 years of age, ODDI $\geq 104 \%$ was established in $17.3 \%$ (35/202) of the no positional preference group and in $34.8 \%(16 / 46)$ of the positional preference groups.

We have to realise that the conclusions have to be considered cautious and could not be generalised, especially not for children in other countries. In The Netherlands, the efforts to reduce positional preference and skull deformation became more and more structural in the last decennia, so it could have influenced the small differences in outcome.

Positional preference at 7 weeks of age seems to be an important determinant of DP in clinical decision making influencing tailored treatment. Therefore, it is necessary to coach parents in handling and stimulating their child, especially in the early months of life and to focus on children with positional preference, which corresponds to the conclusions of the studies by Aarnivala et al. [1] and Cavalier et al. [10]. A short period of PPT is effective in the earlier reduction of DP $[9,43]$, especially when it is started before 3 months of age [44]. This may reduce parental fear and increase self-efficacy. None of the included children got helmet therapy. We did not suggest helmet therapy. Van Wijk et al. discussed the effect of helmet treatment as a conclusion of the HEADS study [45], which had also the practical implication that advises to suggest helmet therapy decreased further.

Hutchison et al. found in their follow-up study of DP cases (measured with the HeadsUp method and almost similar cutoff points) that $39 \%$ did not revert to normal range at 3-4 years of age [20]. Their findings regarding the recovering of DB were comparable with our study findings [20]. Roby et al. found a prevalence of DP in teenagers in a cross-sectional study of $1.1 \%$ and a prevalence of DB of $1.0 \%$ [33], which may suggest further recovery in the following years. This is not in line with our findings on the outcome of DP at 5.5 years and is maybe due to the difference in measuring methods; anthropometric calliper measurements are difficult to compare with PCM and HeadsUp measurements, but this has to be considered as a speculation. Furthermore, differences in the prevalence of skull deformation are probably based on the chosen cut-off points or on the use of different measuring methods for skull deformations.

Future studies have to focus on moderate to severe cases of deformational plagiocephaly (ODDI $\geq 108 \%$ at 7 weeks of age), to advise the parents properly in different stages of skull asymmetry (tailored care). Children with severe and/or progressive skull deformations, which do not recover or stay stable in a typical predictable pattern, deserve special attention and alertness. By starting tailored parent counselling and PPT for a short period in children with persistent positional preference early, most of the initial skull deformations may be avoided [10, 43, 44]. Differential diagnostics are indicated to rule out craniosynostosis, especially in progressive skull deformation and facial asymmetry.

\section{Conclusions}

The course of skull deformation (DP and DB) in newborns is favourable in most children in The Netherlands, especially concerning DB. The deformation recovers to acceptable values in nearly all children at 5.5 years of age. Medical resource consumption may be reduced by providing early tailored parent counselling taking into account natural recovery. One should be alert in those cases where the recovery is not progressing as expected.

Acknowledgments We thank all parents and infants who participated in the study. We thank Wendy Achterberg, Anique Arkink, Loes van den Berg, Anouk Brunninkhuis, Sophieke Charité, Ianthe van der Deijl, Annemarie Duteweerd, Civile Henny, Judith Hukker, Eva Kraaijeveld, Dunja Leeflang, Suzan van Leent, Sofie Nafzger, the paediatric physical therapy students who assisted at the assessments, and also Annette Durieux, Saskia van Exter, Wendy Huurman, Miranda Lahuis, Aletta Pomper, Vivienne Schellekens, Nicole Strikker-Fontijn, Marjolein van Velsen, and Nynke de Zee, the supervising paediatric physical therapists, Femke van Gastel for performing PCM, Annette Roelands for the logistic support, and Lotte van Vlimmeren for the data-entry work. None of these persons received compensation for their contribution.

Authors' contributions Leo van Vlimmeren conceptualised and designed the study, was responsible for the day to day management of the study, acquired, analysed and interpreted the data, drafted the initial manuscript, and approved the final manuscript as submitted. Raoul Engelbert conceptualised and designed the study, interpreted the data, critically reviewed and revised the manuscript, and approved the final manuscript as submitted. Maaike Pelsma analysed and interpreted the data, drafted the manuscript, and approved the final manuscript as submitted. Hans Groenewoud analysed and interpreted the data, drafted the manuscript, 
and approved the final manuscript as submitted. Magda BoereBoonekamp conceptualised and designed the study, interpreted the data, critically reviewed and revised the manuscript, and approved the final manuscript as submitted. Maria Nijhuis-van der Sanden conceptualised and designed the study, analysed and interpreted the data, critically reviewed and revised the manuscript, was the guarantor, and approved the final manuscript as submitted.

All authors had participated sufficiently in the work to take public responsibility for appropriate portions of the content. All authors had full access to all of the data in the study and can take responsibility for the integrity of the data and the accuracy of the data analysis.

Compliance with ethical standards All procedures performed in this study were in accordance with the ethical standards of the institutional and/or national research committee and with the 1964 Helsinki declaration and its later amendments or comparable ethical standards. The Medical Ethics Committees of the University Medical Center Utrecht, The Netherlands (initial part of the study 0-24 months), of the Radboud University Medical Center Nijmegen, The Netherlands (5-year assessments) and of the Bernhoven Hospital Veghel (all assessments), The Netherlands, gave ethical approval. Written informed consent was obtained from all parents of the children in the cohort.

Funding This study was made possible by a grant BU002/10 from the Scientific Committee of The Royal Dutch Association for Physiotherapy, Amersfoort, The Netherlands. There was independence of researchers from funders.

Conflict of interest The authors declare that they have no conflicts of interest. The authors have indicated they have no financial relationships relevant to this article to disclose.

Open Access This article is distributed under the terms of the Creative Commons Attribution 4.0 International License (http:// creativecommons.org/licenses/by/4.0/), which permits unrestricted use, distribution, and reproduction in any medium, provided you give appropriate credit to the original author(s) and the source, provide a link to the Creative Commons license, and indicate if changes were made.

\section{References}

1. Aarnivala H, Vuollo V, Harila V, Heikkinen T, Pirttiniemi P, Valkama AM (2015) Preventing deformational plagiocephaly through parent guidance: a randomized, controlled trial. Eur J Pediatr: Apr [Epub ahead of print]

2. American Academy of Pediatrics. Task Force on Positioning and SIDS (1992) Pediatrics 89:1120-1126

3. American Academy of Pediatrics. Task Force on Sudden Infant Death Syndrome (2011) SIDS and other sleep-related infant deaths: expansion of recommendations for a safe infant sleeping environment. Pediatrics 128:e1341

4. Argenta LC, Davis LR, Wilson JA, Bell WO (1996) An increase in infant cranial deformity with supine sleeping position. J Craniofacial Surg 7:5-11

5. Bialocerkowski AE, Vladusic SL, Howell SM (2005) Conservative interventions for positional plagiocephaly: a systematic review. Dev Med Child Neurol 47:563-570

6. Bialocerkowski AE, Vladusic SL, Wei Ng C (2008) Prevalence, risk factors, and natural history of positional plagiocephaly: a systematic review. Dev Med Child Neurol 50(8):577-586
7. Boere-Boonekamp MM, van der Linden-Kuiper AT (2001) Positional preference: prevalence in infants and follow-up after two years. Pediatrics 107:339-343

8. Brand PL, Engelbert RH, Helders PJ, Offringa M (2005) Systematic review of the effects of therapy in infants with the KISS syndrome (kinetic imbalance due to suboccipital strain). Ned Tijdschr Geneeskd 149(13):703-707

9. Cabrera-Martos I, Valenza MC, Benítez-Feliponi A, RoblesVizcaíno C, Ruiz-Extremera A, Valenza-Demet G (2013) Clinical profile and evolution of infants with deformational plagiocephaly included in a conservative treatment program. Childs Nerv Syst 29(10):1893-1898

10. Cavalier A, Picot M-C, Artiaga C, Mazurier E, Amilhau M-O, Froye E, Captier G, Picaud J-C (2011) Prevention of deformational plagiocephaly in neonates. Early Hum Dev 87:537-543

11. Collett B, Breiger D, King D, Cunningham M, Speltz M (2005) Neurodevelopmental implications of deformational plagiocephaly. J Dev Behav Pediatr 26:379-389

12. de Jonge GA, Engelberts AC, Koomen-Liefting AJM, Kostense PJ (1989) Cot death and prone sleeping position in the Netherlands. BMJ 298:722

13. Dwyer T, Ponsonby A-L, Newman NM, Gibbons LE (1991) Prospective cohort study of prone sleeping position and sudden infant death syndrome. Lancet 837:1244-1247

14. Engelberts AC, de Jonge GA (1990) Choice of sleeping position for infants: possible association with cot death. Arch Dis Child 65:462467

15. Fleming PJ, Gilbert R, Azaz Y (1990) Interaction between bedding and sleeping position in the sudden infant death syndrome: a population based case-control study. BMJ 301:85-89

16. Fong BF, Savelsbergh GJ, van Geijn HP, de Vries JI (2005) Does intra-uterine environment influence foetal head-position preference? A comparison between breech and cephalic presentation. Early Hum Dev 81(6):507-517

17. Golden KA, Beals SP, Littlefield TR, Pomatto JK (1999) Sternomastoid imbalance versus congenital muscular torticollis: their relationship to positional plagiocephaly. Cleft PalateCraniofacial J 36:256-261

18. Graham JM, Gomez M, Halberg A, Earl DL, Kreutzman JT, Cui J, Guo X (2005) Management of deformational plagiocephaly: repositioning versus orthotic therapy. J Pediatr 146:258-262

19. Hutchison BL, Hutchison LA, Thompson JM, Mitchell EA (2004) Plagiocephaly and brachycephaly in the first two years of life: a prospective cohort study. Pediatrics 114:970-980

20. Hutchison BL, Stewart AW, Mitchell EA (2011) Deformational plagiocephaly: a follow-up of head shape, parental concern and neurodevelopment at ages 3 and 4 years. Arch Dis Child 96:85-90

21. Joganic JL, Lynch JM, Littlefield TR, Verrelli BC (2009) Risk factors associated with deformational plagiocephaly. Pediatrics 124:e1126-e1133

22. Johns FR, Jane JA Sr, Lin KY (2000) Surgical approach to posterior skull deformity. Neurosurg Focus 9(3):e4

23. Kaplan SL, Coulter C, Fetters L (2013) Physical therapy management of congenital muscular torticollis: an evidence-based clinical practice guideline. Pediatr Phys Ther 25:348-394

24. Kluba S, Kraut W, Reinert S, Krimmel M (2011) What is the optimal time to start helmet therapy in positional plagiocephaly? Plast Reconstr Surg 128(2):492-498

25. Lekovic GP, Baker BJ, Lekovic JM, Preul MC (2007) New world cranial deformation practices: historical implications for pathophysiology of cognitive impairment in deformational plagiocephaly. Neurosurg 60:1137-1147

26. Littlefield TR, Kelly KM, Pomatto JK, Beals SP (2002) Multiplebirth infants at higher risk for development of deformational plagiocephaly: II. Is one twin at greater risk? Pediatrics 109:9-25 
27. McGarry A, Dixon MT, Greig RJ, Hamilton DRL, Sexton S, Smart H (2008) Head shape measurement standards and cranial orthoses in the treatment of infants with deformational plagiocephaly: a systematic review. Dev Med Child Neurol 50(8):568-576

28. Meyer-Marcotty P, Böhm H, Linz C, Kochel J, Blecher C, Keil N, Stellzig-Eisenhauer A, Schweitzer T (2014) Spectrum of positional deformities - is there a real difference between plagiocephaly and brachycephaly? J Craniomaxillofac Surg 42(6):1010-1016

29. Peitsch WK, Keefer CH, LaBrie RA, Mulliken JB (2002) Incidence of cranial asymmetry in healthy newborns. Pediatrics 10:e72

30. Persing J, James H, Swanson J, Kattwinkel J (2003) Prevention and management of positional skull deformities in infants. American Academy of Pediatrics Committee on practice and ambulatory medicine, section on plastic surgery and section on neurological surgery. Pediatrics 112(1 Pt 1):199-202

31. Pomatto JK, Calcaterra J, Kelly KM, Beals SP, Manwaring KH, Littlefield TR (2006) A study of family head shape: environment alters cranial shape. Clin Pediatr 45:55-63

32. Robinson S, Proctor M (2009) Diagnosis and management of deformational plagiocephaly. A review. J Neurosurg Pediatr 3:284-295

33. Roby BB, Finkelstein M, Tibesar RJ, Sidman JD (2012) Prevalence of positional plagiocephaly in teens born after the "back to sleep" campaign. Otolaryngol Head Neck Surg 146(5):823-828

34. Rogers GF (2011) Deformational plagiocephaly, brachycephaly, and scaphocephaly. Part I: terminology, diagnosis, and etiopathogenesis. J Craniofac Surg 22:9-16

35. Sergueef N, Nelson KE, Glonek T (2006) Palpatory diagnosis of plagiocephaly. Complement Ther Clin Pract 12(2):101-110

36. Seruya M, Oh AK, Taylor JH, Sauerhammer TM, Rogers GF (2013) Helmet treatment of deformational plagiocephaly: the relationship between age at initiation and rate of correction. Plast Reconstr Surg 131(1):55e-61e

37. Teichgraeber JF, Seymour-Dempsey K, Baumgartner JE, Xia JJ, Waller AL, Gateno J (2004) Moulding helmet therapy in the treatment of brachycephaly and plagiocephaly. J Craniofac Surg 15(1): $118-123$
38. van Adrichem LNA, van Vlimmeren LA, Cadanová D, Helders PJM, Engelbert RHH, van Neck HJW, Koning HJ (2008) Validation of a simple method for measuring cranial deformities (plagiocephalometry). J Craniofac Surg 19(1):15-21

39. van Vlimmeren LA, Helders PJ, van Adrichem LN, Engelbert RH (2004) Diagnostic strategies for the evaluation of asymmetry in infancy: a review. Eur J Pediatr 163(4-5):185-191

40. van Vlimmeren LA, Helders PJ, van Adrichem LN, Engelbert RH (2006a) Torticollis and plagiocephaly in infancy: therapeutic strategies. Pediatr Rehabil 9(1):40-46

41. van Vlimmeren LA, Takken T, van Adrichem LN, van der Graaf Y, Helders PJ, Engelbert RH (2006b) Plagiocephalometry: a noninvasive method to quantify asymmetry of the skull: a reliability study. Eur J Pediatr 165:149-157

42. van Vlimmeren LA, van der Graaf Y, Boere-Boonekamp MM, L'Hoir MP, Helders PJM, Engelbert RHH (2007) Risk factors for deformational plagiocephaly at birth and at seven weeks of age. A prospective cohort study Pediatrics 119:e408-e408

43. van Vlimmeren LA, van der Graaf Y, Boere-Boonekamp MM, L'Hoir MP, Helders PJM, Engelbert RHH (2008) Paediatric physical therapy is effective in reducing deformational plagiocephaly in children. A randomized controlled trial. Arch Pediatr Adolesc Med 162:712-718

44. van Wijk RM, Pelsma M, Groothuis-Oudshoorn CG, IJzerman MJ, van Vlimmeren LA, Boere-Boonekamp MM (2014a) Response to paediatric physical therapy in infants with positional preference and skull deformation. Phys Ther 94(9):1262-1271

45. van Wijk RM, van Vlimmeren LA, Groothuis-Oudshoorn CGM, van der Ploeg CPB, IJzerman MJ, Boere-Boonekamp MM (2014b) Helmet therapy in infants with positional skull deformation: randomised controlled trial. BMJ 348:g2741

46. Xia JJ, Kennedy KA, Teichgraeber JF, Wu KQ, Baumgartner JB, Gateno J (2008) Nonsurgical treatment of deformational plagiocephaly: a systematic review. Arch Pediatr Adolesc Med 162(8):719-727 